\title{
CRISES FOCAIS NA HIPERGLICEMIA NAO CETÓTICA
}

\author{
CARLOS A. M. GUERREIRO * - ANTONIO E. FALCÃO ** \\ DIOSELY C. SILVEIRA ***
}

\begin{abstract}
RESUMO - Os casos de três pacientes com crises focais associadas a hiperglicemia não cetótica (HNC) são relatados. Os dois primeiros tiveram epilepsia parcial contínua (EPC) motora. No primeiro paciente a EPC foi a manifestação inicial do diabetes mellitus. O exame neurológico mostrou-se normal em todos os pacientes. TC de crânio e exame do LCR foram normais, quando realizados. O EEG, durante a EPC em um caso, mostrou atividade critica epileptiforme nas regiões temporais bilateralmente, a despeito da manifestação clínica ser unilateral. Os níveis glicêmicos foram de 455, 660 e $439 \mathrm{mg} / \mathrm{dl}$. Dois pacientes apresentavam hiponatremia concomitante. Nenhum dos pacientes respondeu à hidantalização ou ao uso de benzodiazepínicos; em um deles houve acentuação das crises após sua administração. O controle das crises só ocorreu com insulinoterapia e correção hidroeletrolítica. O reconhecimento da HCN como causa de EPC, sem lesão estrutural evidenciável, é de grande importância no sentido de orientar a terapêutica adequada para o controle das crises e de evitar evolução para síndrome de HNC com hiperosmolaridade e alteração de consciência, o que leva a mortalidade significante.
\end{abstract}

Partial seizures in non-cetotic hyperglycemia.

SUMMARY - The cases of three patients with focal seizure associated to non-cetotic hyperglycemia are reported. Two patients presented motor epilepsy partialis continua (EPC). One case showed EPC as the first clinical manifestation of diabetes mellitus. Neurological exam was normal in all patients. CT and CSF were normal in the cases they were evaluated. Scalp EEG registered during a focal seizure revealed a bilateral temporal spiky activity. Glycemia levels were 455, 660 and 439mg/dl. Two patients presented hyponatremia simultaneously. No patiets had beneficit with phenytoin or diazepan, and one patient got worse after them. Seizure control occurred after insulin and electrolytic treatment. It is important to diagnose this type of condition to avoid changes of non-cetotic hyperglycemia syndrome in a hypero3molarity and coma state, disturbance which brings a higher mortality.

Diabetes mellitus com hiperglicemia sem cetose pode estar presente por meses antes que a doença se torne clara ${ }^{3}$. Epilepsia focal como manifestação clínica da hiperglicemia não cetótica (HNC) tem sido descrita desde 19651.8. Manifestações epilépticas focais no curso da HNC ocorrem em torno de $19 \%$ dos casos e constituem o sintoma inicial, precedendo a alteração de consciência, em torno de $6 \%{ }^{4}$.

Relatamos os casos de três pacientes que apresentaram crises epilépticas focais associadas a HiNC e discutimos o tema à luz da literatura disponível.

Departamento de Neurologia, Faculdade de Ciências Médicas (FCM), Universidade Estadual de Campinas (UNICAMP): * Professor Assistente Doutor; ** Médico, Hospital das Clí-ᄀ nicas/UNICAMP; *** Professor Assistente.

Dr. Carlos Alberto Mantovani Guerreiro - Departamento de Neurologia, Faculdade de Ciências Médicas, UNICAMP - Caixa Postal 6111 - 18081 Campinas SP - Brasil. 


\section{CASUística}

Caso 1 - RMC, paciente com 62 anos de idade, do sexo masculino, químico industrial, iniciou quadro de movimentos clónicos e/ou tônicos no membro superior (MS) esquerdo (E) após almoço. Cada episódio durava aproximadamente 30 minutos e não deixava déficit. As crises ocorriam principalmente durante o dia, porém eventualmente chegando a acordar o paciente durante a noite. Exames laboratoriais realizados na empresa em que trabalha, aproximadamente um mês antes do início do quadro, estavam normais, inclusive glicemia de jejum. Eletrencefalograma (EEG) crítico mostrou atividade epileptiforme temporal bilateral, apesar de ser a crise unilateral. A tomografia computadorizada craniana (TC) mostrou-se normal. Foi administrada fenitoína, após o que foi notada piora das crises, acometendo os membros de forma isolada e generalizada. Diagnosticado diabetes mellitus, com: glicemia $455 \mathrm{mg} / \mathrm{dl}$, sódio $128 \mathrm{meq} / 1$, potássio $3,2 \mathrm{meq} / 1$. Suspensa a fenitoína, foi tratado com dieta, tendo as crises desaparecido em 24 horas. Seguido há mais de três anos, apenas com dieta para diabéticos, permanece assintomático.

Caso 2 - MM, paciente com 63 anos de idade, do sexo feminino, diabética em uso de hipoglicemiante oral. Após uso abusivo de refrigerantes não dietéticos, apresentou confusão mental e desvio clónico da rima bucal para direita (D). Depois de vários episódios as crises motoras desapareceram, com alteração no nível de consciência. Apresentava, na entrada do pronto socorro: glicemia $660 \mathrm{mg} / \mathrm{dl}$, sódio $132 \mathrm{meq} / 1$ e potássio $3,8 \mathrm{meq} / 1$. Após correção da glicemia, os sintomas neurológicos desapareceram e a paciente evoluiu assintomática por, pelo menos, há um ano.

Caso 3 - LCR, paciente com 36 anos de idade, do sexo masculino, diabético, apresentou cefaléia de leve intensidade na região parietal $D$ seguida, dois dias depois, de crises parciais simples sensitivo-motoras à E. Fazia uso regular de insulina NPH 30 u/dia. Ao exame, estava consciente, orientado, sem sinais neurológicos focais. Líquido cefalorraquidiano (LCR) normal, a exceção de hiperglicorraquia. TC revelou-se normal. EEG mostrou ondas lentas intermitentes na região temporal D. Glicemia $439 \mathrm{mg} / \mathrm{dl}$, sódio $137 \mathrm{meq} / 1$ e potássio 4,3 meq/1. Introduzida fenitoína, houve acentuação das crises (até 2 a 3 por dia). A dose de insulina foi aumentada para 50 u NPH e foi dada orientação dietética. Após 6 dias de internação, com o controle da glicemia o paciente ficou sem crises epilépticas e sem medicação anticonvulsivante.

\section{COMENTÁRIOS}

A epilepsia focal, como manifestação de HNC, foi primeiro observada em 1965 por Maccario et alA Posteriormente, foram relatados 5 casos de epilepsia parcial contínua (EPC) e HNC por Sing et al.6 que observaram poder a EPC ser o sintoma inicial em pacientes alertas. Os mesmos autores, em 1980, relataram 21 casos de HNC que se apresentaram com EPC ${ }^{7}$. Em 1985, Grant e Warlo ${ }^{3}$ relataram os casos Je 5 pacientes que tinham crises focais de início tardio, sem história prévia de epilepsia, acidente vascular cerebral ou trauma craniano. Nenhum deles apresentava lesão cerebral focal detectável e, em todos, a epilepsia focal motora foi o primeiro sintoma do diabetes.

Nenhum dos nossos pacientes tinha história prévia de trauma cranioencefálico, acidente vascular cerebral, ou história familiar de epilepsia. A TC mostrou-se normal nos casos 1 e 3 . Todos apresentaram epilepsia focal motora como manifestação da hiperglicemia, no primeiro caso sendo manifestação inicial do diabetes. Em todos os pacientes o exame neurológico mostrou-se normal e o principal passo terapêutico no controle das crises foi a correção glicêmica.

A patogênese da crise focal na HNC não está bem esclarecida. Acredita-se que seja o resultado da ativação de disfunção focal anteriormente silenciosa (lesão isquémica), pela hiperglicemia e/ou hiperosmolaridade. Sing e Strobos? encontraram, em 15 de 21 pacientes, hiponatremia concomitante e levantaram a possibilidade de colabotar a hiponatremia no desencadeamento das crises epilépticas nesses pacientes.

Curiosamente, encontramos pacientes nos quais as crises foram a primeira manifestação do diabetes, como no caso 1. Desta maneira, é fundamental a solicitação de glicemia na investigação de pacientes com manifestação epiléptica focal de início agudo, independente de história prévia de diabetes. $O$ reconhecimento precoce da hiperglicemia como causa de HNC sem lesão estrutural evidenciável é de grande importância no sentido de orientar a terapêutica adequada para o controle das crises e de evitar evolução para a síndrome de HNC com hiperosmolaridade e alteração do nível de consciência, o que leva a mortalidade significativa. 


\section{REFERENCIAS}

1. Askenasy JJ, Streifler M, Carrasco R. Moderate nonketotic hyperglycemia: a cause of focal epilepsy. Eur Neurol 1977, 16 : 51-61.

2 Daniels CJ, Chokroverty S, Barron KD. Anacidotic hiperglycemia and focal seizures Arch Intern Med 1969, 124 : 701-706.

3. Grant C, Warlow C. Focal epilepsy in non-ketotic hiperglycemia. Br Med J 1985,290 1204-1205.

4. Graveleau F, Martigny R, Dairou R, Masson M, Graveleau J. Epilepsie motrice partielle au cours d'une hyperglycémie non cétosique. Presse Méd 1984, 13 : 1751-1752.

5. Maccario M, Messis CP, Vastola EF - Focal seizures as a manifestation of hyperglycemia without ketoacidosis. Neurology 1965, 15 : 195-206.

6. Sing BM, Grupta DR, Strobos RJ. Nonketotic hyperglycemia and epilepsia partialis continua. Arch Neurol 1973, 29 : 187-190.

7. Sing BN, Strobos RJ. Epilepsia partialis continua associated with nonketotic hyperglycemia: clinical and biochemical profile of 21 patients. Ann Neurol 1980, 8:155-160.

8. Venna N, Sabin TD. Tonic focal seizures in nonketotic hyperglycemia of diabetes mellitus Arch Neurol 1981, 38 : 512-514. 